“ (C) 2013 IEEE. Personal use of this material is permitted. Permission from IEEE must be obtained for all other uses, in any current or future media, including

reprinting/republishing this material for advertising or promotional purposes, creating new collective works, for resale or redistribution to servers or lists, or reuse of any copyrighted component of this work in other works." 


\section{Development of a Novel Evidence-Based Automated Powered Mobility Device Competency Assessment}

\author{
Jaime Valls Miro \\ Faculty of Engineering and IT \\ University of Technology Sydney (UTS) \\ Sydney NSW 2007, Australia \\ jaime.vallsmiro@uts.edu.au \\ Ross Black \\ Occupational Therapy Department \\ Prince of Wales Hospital \\ Sydney NSW 2031 \\ ross.black@sesiahs.health.nsw.gov.au
}

\author{
Bojan Andonovski and Gamini Dissanayake \\ Faculty of Engineering and IT \\ University of Technology Sydney (UTS) \\ Sydney NSW 2007, Australia \\ bojan.andonovski@student.uts.edu.au \\ gamini.dissanayake@uts.edu.au
}

\begin{abstract}
This paper describes the outcomes of a clinical study to assess the validity of a stand-alone sensor package and algorithms to aid the assessment by an occupational therapist (OT) whether a person has the capacity to safely and effectively operate a powered mobility device such as a wheelchair in their daily activities. The proposed solution consists of a suite of sensors capable of inferring navigational characteristics from the platform it is attached to (e.g. trajectories, map of surroundings, speeds, distance to doors, etc). Such information presents occupational therapists with the ability to augment their own observations and assessments with correlated, quantitative, evidence-based data acquired with the sensor array. Furthermore, OT reviews can take place at the therapist's discretion as the data from the trials is logged. Results from a clinical evaluation of the proposed approach, taking as reference the commonly-used Power-Mobility Indoor Driving Assessment (PIDA) assessment, were conducted at the premises of the Prince of Wales (PoW) Hospital in Sydney by four users, showing consistency with the OT scores, and setting the scene to a larger study with wider targeted participation.
\end{abstract}

\section{INTRODUCTION}

Powered mobility devices (PMDs) such as electric wheelchairs and scooters are some of the most popular ambulation devices used by the aged and disabled population. The benefit they bring in maximising the independence of people in the community can be so prominent they are sometimes regarded in equal terms to the rehabilitation treatment programs [1]. Adults aged over 50 years are the most prevalent wheelchairs users [2], and it is estimated that PMD use is 3.5 times more frequent after the age of 65 years [3].

Matching the technology of PMDs to user's needs is a long and complex process. The devices are heavy and fast-moving and can be used both in- and out-doors in the presence of static (architectural barriers) and dynamic (pedestrian and vehicular traffic) hazards. In a typical PMD assessment a therapist will observe the client using the device in the environments in which it is to be used, and take into consideration a number of factors when prescribing the use of a specific wheelchair (e.g. user's experience, motor, sensory, vestibular, cognitive and visual skills, the terrain in which the device is to be used, device components - seating arrangements [4], drive configuration, controllers etc).

While there are a number of tools used by OTs to assess user acceptance of technology in general, these are often subjective: the Psychosocial Impact of Assistive Devices (PIADS), the Occupational Therapy Functional Assessment Compilation Tool (OTFACT), the Assistive Technology Outcome Measure (ATOM) or the Wheelchair Outcome Measure (WhOM)) and not necessarily specific to the device being assessed [5]. Some wheelchair user-specific questionnaires have also been developed to assess self-perceived wheelchair skills e.g. the Wheelchair Skills Test (WST) [6](revised [7]), and functions related to wheelchair/scooter use e.g. the Functional Evaluation in a Wheelchair [8], Power-Mobility Indoor Driving Assessment (PIDA) [9] and the Power-Mobility Community Driving Assessment (PCDA) [10]. The WST is applicable to manual and powered wheelchair (WST-P) users and has undergone quite rigorous validation procedures [11]. The test has become the preferred method of assessing a users ability to use a manual wheelchair and has even been described as the gold standard in research and clinical areas [11], [12]. The Functional Evaluation in a Wheelchair Questionnaire (FEWQ) [8] and the Obstacle Course Assessment of Wheelchair User Performance (OCAWUP) [13] are also employed to assess PMD use. The FEW-Q focuses on the interaction between the wheelchair user, the technology of the wheelchair and the environments of use. The OCAWUP is designed to identify obstacles that a wheelchair user is likely to encounter in daily life and their social roles. Many organizations and service providers have also developed their own check-list of parameters to be assessed e.g. the Department of Veterans' Affairs "EWC_Scooter Guidelines" 2010 or the "Occupational Therapy Power Wheelchair Assessment". Recently, several authors have used accelerometers mounted on manual wheelchairs to determine the users velocity, distance travelled, activity patterns and bouts of mobility during activities of daily living (ADLs) [14], [15]. Arm and trunk wheelchair mounted accelerometers have also been used to measure the physical activity levels of wheelchair users [16]. The technology is cheap, reliable and can be applied to almost any mobility platform. Other sensorial data, such as that measured directly from the standard wheelchair joystick controller, has been used to differentiate novice and expert electric wheelchair users [17], whilst exteroceptive sensors such as cameras and laser range finders have also been proposed to determine task metrics of safety, smoothness, directness and global efficiency in order to develop a wheelchair-user collaborative control system for indoor navigation [18].

Generally, the models of practice and assessments used for the selection of assistive technology are poorly developed and concerns have been raised about the set of available 
wheelchair assessments, clearly indicating the need for further research. An extensive review of the literature from 20032007 concluded that there is lack of evidence-based procedures for the selection of assistive technology [19]. The need for higher levels of evidence-based practice was concluded in relation to matching of mobility assistive technology in people with multiple sclerosis [20]. Large inconsistencies have been reported between currently available manual wheelchair tests [21]. Also, commonly used standarized PMD assessments are not intended to determine whether or not a person will be a safe driver and don't assist therapists in determining when risk becomes untenable [22]. But maybe more relevantly in recent times, funding bodies in countries such as Australia are increasingly requiring more detailed and specific data about the intended uses and suitability of expensive pieces of equipment e.g. electric wheelchairs and scooters, before they will support therapist's applications for these types of equipment.

There is clearly a pressing need to augment the existing therapist's subjective assessments of PMDs use with more objective and quantitative performance indicators. The use of subjective and objective assessments would provide complementary, but distinct, information allowing a more complete assessment of mobility [23]. This is an important observation: quantitative assessment does not mean a preference over subjective/qualitative assessment. There is a need to "complement", not to replace, one with the other. Therapists are highly skilled at combining observations with evaluation, being trained to see behaviour but judge the quality of it simultaneously. Yet the additional data can be interpreted by the therapists and used to support and augment the therapist's observations in completing a comprehensive evidence-based evaluation of a person's PMD use. This, in turn, presents occupational therapists (OTs) with the capability to factually decide whether patients are ready to be safely deployed with mobile aids for their daily activities.

This paper describes the results of a clinical study where OT's scores drawn from a sub-set of those commonly used in an indoor wheelchair assessment (PIDA) are compared to a range of objective parameters (described in Section IV) inferred from the measured wheelchair navigational data. The solution encompasses a standalone package of sensing and computing (described in Section II) able to be deployed on a standard mobile platform, such as a wheelchair or a walker, and a suite of algorithms (described in Sections III and IV) capable of generating a map of the surrounding environment and estimating the trajectory - and associated navigational parameters - followed by the wheeled platform while performing the set of given tasks in the assessment. Good consistency between the subjective scores and objective data, as revealed in Section V, points at the the validity of employing a system which allows therapists to complement their evaluations with additional objective data about environments of use and operation of PMDs in those environments, as will be discussed in Section VI.

\section{Navigational Sensor Package}

The proposed sensor package is a small size, light-weight, self-contained package that can be easily mounted on most mobility aids with standard fixtures. In this work, the package was mounted on a standard Pride LX electric wheelchair,

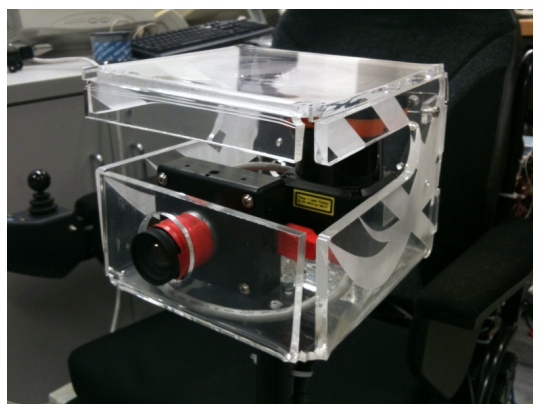

(a) Sensor package prototype - detail.

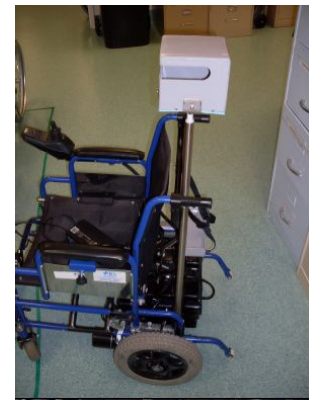

(b) Sensor enclosure mounted on wheelchair
Fig. 1. Sensor package - detail and enclosure as mounted on a standard electric wheelchair at the PoW hospital.

one of the platforms regularly used by the OTs at the PoW hospital in their routine assessments, and will also be used in the experiments presented in this paper. The approximate total weight for the sensor package enclosure is less than $1 \mathrm{Kg}$, i.e. safe to handle and easy to place in secured high-centered positions for a wider field of view, as is the case in the PoW hospital wheelchair. The sensor package employed consists of:

- A Hokuyo UTM-30LX/LN scanning laser range finder, able to measure distance to objects between $\{0.1 \mathrm{~m}-30 \mathrm{~m}\}$ in a semicircular field of $270^{\circ}$.

- A Point Grey Dragonfly2 Firewire camera able to capture high resolution (1032x776pixels) colour images at $30 f$ s.

- A Xsens MTi inertial measurement unit (IMU), a low weight $3 \mathrm{DoF}$ attitude and heading reference system capable of measuring accelerations, angular velocities and magnetic orientations.

A detail picture of the standalone enclosure depicting the configuration of the sensors being displayed in Figure 1a. Based on this set-up, the actual enclosure designed to the requirements of the Biomedical Engineering department at the PoW hospital for deployment within the hospital grounds can be seen in Figure 1b. For further details about the sensor design and capabilities the reader is referred to [24].

\section{MAPPING AND WHEELCHAIR TRACKING}

The first step in the proposed process of quantitative assessment is that of mapping the surroundings and tracking the PoW hospital wheelchair platform with the sensor array mounted on it. Data was logged while the wheelchair was driven around by users in an indoor hospital test area, as will be described later in Section V. Two-dimensional (topdown view) maps of the environment, like the ones depicted in Figure 2, could then be generated from the laser range finder and IMU data collected. Logged streaming data was not used for navigational purposes in this study, but it allowed the OT to visually replay the user runs at a latter date for further study. The software framework employed in this work is built around ROS (www.ros.org), an open source meta-operating system middleware for robotic platforms. Amongst other things, it provides a range of tools to map and localize mobile robotic 


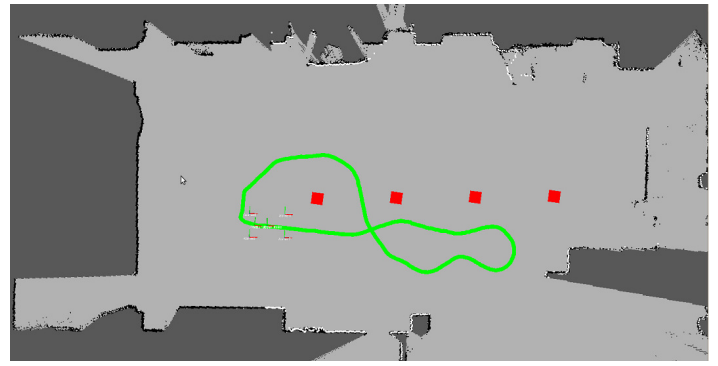

(a) Wheelchair trajectory while performing Task 1 run (user 4)

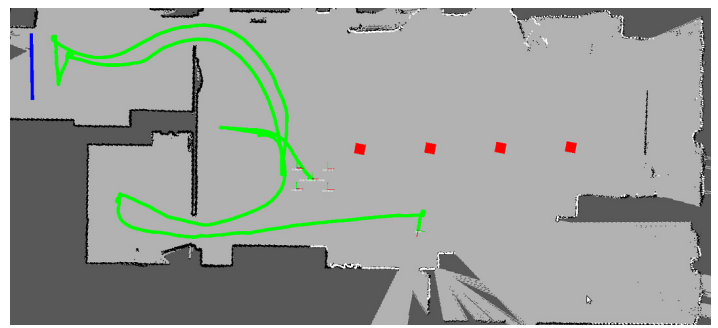

(c) Wheelchair trajectory while performing Task 3 run (user 4)

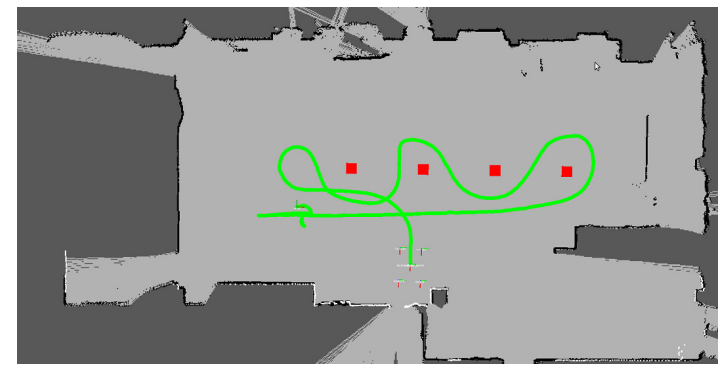

(b) Wheelchair trajectory while performing Task 2 run (user 4)

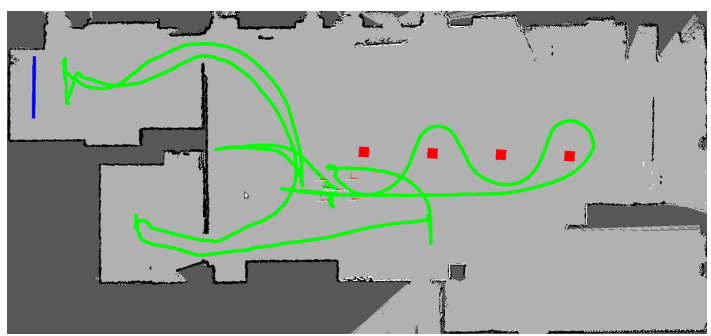

(d) Wheelchair trajectory while performing the final Task 4 run (user 4) - a combination of Tasks 1, 2 and 3

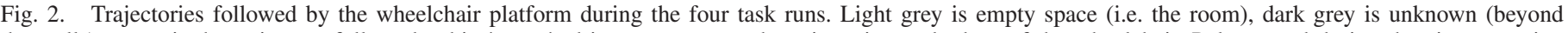

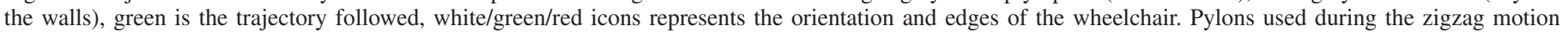
are shown in red, while the right edge of the bed in the left room is shown in blue.

platforms as they move about based on sensorial feedback from the environment, as was described in our earlier work [24].

For this approach to operate successfully both laser range data and wheelchair odometry are generally needed. However this can only be possible for customized platforms where wheel odometers have been fitted and interfaced with. On the other hand, a key feature of the proposed strategy is that of portability, so that the package can be easily fit to any mobility aid without further instrumentation. In our previous work [24] we made use of a 2D SLAM (Simultaneous Localization and Mapping) [25] algorithm based on a Rao-Blackwellized particle filter were odometry was virtually generated, effectively learning occupancy grid maps from the 2D laser range data [26]. The generated maps were then re-used to localize the platform in the learned environment producing the paths followed by the wheelchair [27]. However, this solution has got a number of drawbacks: it works best in fully planar environments, do not leverage the high update rate provided by modern sensors systems (such as laser range finders), and still rely on sufficiently accurate odometry being estimated. The end result are wheelchair trajectories highly sensitivity to correct parameter settings, which impact negatively in the additional navigational metrics (described in SectionIV below) proposed in this work to complete an objective assessment of the wheelchair user's driving abilities.

To that end, this work suggests an alternative SLAM proposition where instead of generating virtual odometry through scan-matching, it operates on the principle of integrating laser scans in a planar map. As scans get aligned with the existing map, the matching is implicitly performed with all preceding scans. The system is accurate enough not to need explicit loop-closure to attain accurate trajectory information in many real world scenarios, as well as being better suited for fast online learning of occupancy grid maps requiring low computational resources. It combines a robust laser scan matching approach [28] with 3D attitude estimation from the on-board inertial sensing. By using a fast approximation of map gradients and a multi-resolution grid, reliable localization and mapping capabilities in a variety of challenging environments have been reported in the literature [29]. It also integrates into the API of the the ROS navigation stack, and thus can be easily interchanged with other SLAM approaches or other probabilistic localisations modules available in the ROS ecosystem.

\section{Additional NaVigational Parameters}

After the maps were created, and the paths followed by the platform during the trials estimated, it is now clearly possible for therapists and clinicians to examine other navigational parameters that can help them to obtain a qualitative assessment of the user's driving ability.

While there are a considerable number of quantitative registrations that can be measured from the inferred trajectory, this work has focused on four key metrics so that the comparison with the OT's qualitative PIDA scoring can be more effectively carried out:

1) alignment with beds (e.g. Figure 3a)

2) proximity to doors (e.g. Figure $3 b$ )

3) linear velocity profile (e.g. Figure 4)

4) angular velocity profiles (e.g. Figure 5)

When accessing the bed from the right side, as in our test set up, the PIDA instructions call for the client to manoeuvre 


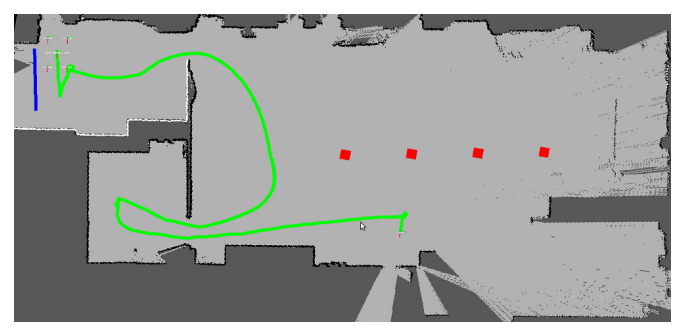

(a) Bed alignment sub-task

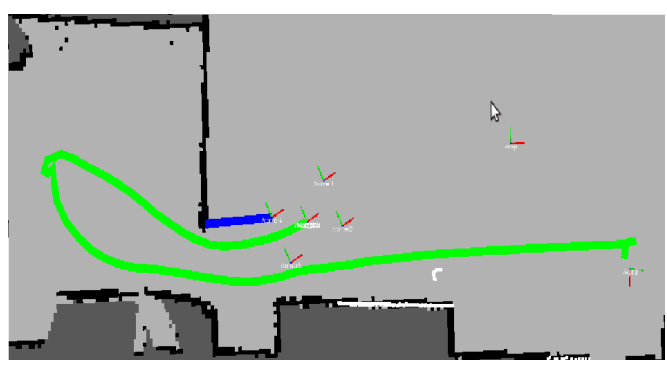

(b) Driving through door sub-task, with minimum distance vector at this instant shown in blue.

Fig. 3. Visualization of the bed-alignment (left) and drive through door metric extraction, both components of Task 3

on the right side of the bed so that they can move directly to the bed. We used the distance and orientation to the bed information to analyse the user's ability to manoeuvre the wheelchair to access the bed for a transfer. Similarly, distance to door is not specifically assessed in the PIDA but is a factor in determining if a user is likely to bump into the door and cause harm to themselves, other people or objects. Our assumption was that the OT would possibly use these parmeters in their assessment and we wished to see if this was the case. If so, there would be a correlation between the objective and subjective scores. The OT was not specifically required to assess these paparmeters. Speed is an important observation that can be used to infer a user's confidence and situational awareness. When crossing the road a wheelchair user will need to proceed quickly. However, when surrounded by other people or when approaching a doorway speed will need to be reduced. The parameters derived from the sensor package measurements may allow for instance the assessing team/therapist to determine if a wheelchair user was able to select the speed appropriate to the environment of use, and to accurately record and analyse this parameter as the person spends more time using the device. Similarly, as will be seen in the experimental Section V, if a wheelchair user's speed profile was found to be low, as compared with that of a skilled user in a particular environment, it might be used to indicate the need to adjust parameters on the chair such as seating or position of the wheelchair controller. On the other hand, if the wheelchair was properly set up, such behaviour might suggest lack of confidence or the impact of any visual or cognitive impairment in a user and the need for further input by the therapist.

Other parameters (e.g. distance to a wall a user might be requested to follow, acceleration, idle time, etc.) can also be inferred but are left for a future study. The choice of parameters is limited only by the requirements of the assessing

\begin{tabular}{|c||c|c||c|c|c|}
\hline User & $\begin{array}{c}\text { Distance to } \\
\text { door }(\mathrm{m})\end{array}$ & OT's score & $\begin{array}{c}\text { Orientation to } \\
\text { bed }\left({ }^{\circ}\right)\end{array}$ & $\begin{array}{c}\text { Distance to } \\
\text { bed }(\mathrm{m})\end{array}$ & OT score \\
\hline \hline 1 & 0.13 & 4 & 1 & 0.55 & 3 \\
\hline 2 & 0.14 & 3 & 5 & 0.73 & 3 \\
\hline 3 & 0.13 & 3 & 18 & 0.46 & 2 \\
\hline 4 & 0.14 & 4 & 6 & 0.61 & 3 \\
\hline
\end{tabular}

TABLE I. SPECIFIC USER PERFORMANCE METRICS AND OT SCORES DURING THE "ADVANCE TASK 3".

team/therapist.

\section{EXPERIMENTAL EVALUATIONS}

The PIDA wheelchair assessment was chosen to evaluate the proposed approach. This is the routine commonly use at the PoW facility where the field trials were conducted, hence is also more familiar to the OT who carried out the manual assessments. Performances on test subections are scored from 1 to 4. Optimal performance recieves a score of "4" and inability to complete a task would be given a score of "1". Multiple or hesitant attempts or those in which a user bumps into objects or people would be rated "2" or "3" depending on the liklihood of harm to the user, people or other objects. Four able users of differing abilities in operating electric wheelchairs were asked to perform four runs each representing a range of tasks broadly aligned to those outlined in the PIDA assessment. For reference, these tasks have been categorized into "familiarization" (task 1), "basic" (task 2) and "advanced" (task 3) ${ }^{1}$ :

- $\quad$ Task 1 (Getting familiar)

- Warm up task to get familiar with the chair. No specific tasks given.

- $\quad$ Task 2 (Basic control)

- Turn $90^{\circ}$ left and right on the spot.

- Drive $5 \mathrm{~m}$ in a straight line.

- Drive the wheelchair in a zig-zag pattern around 5 pylons spaced at $1.5 \mathrm{~m}$.

- Drive the wheelchair towards a closed door without driving into it, here the person driving the chair should be able to open the door.

- $\quad$ Task 3 (Advanced control)

- Drive the wheelchair through a doorway without touching the sides.

- Drive the wheelchair underneath a table, so the person driving the chair can interact with objects on the table.

- Position the wheelchair so it is oriented sideways to a bed in preparation for a sliding-board transfer. The person should be able to touch the pillow of the bed. Ideally, the chair is parallel to the bed and as close as possible.

- Back the wheelchair between two tables and keep the chair parallel to the tables.

The trajectories shown in Fig. 2, which correspond to user 4, are representative of the various tasks described above.

\footnotetext{
${ }^{1}$ An additional Task 4 , combining all previous 3 in a single, sequential run, was also carried out, although could not be completed by all 4 users for logistic reasons (only 2 users could do it)
} 


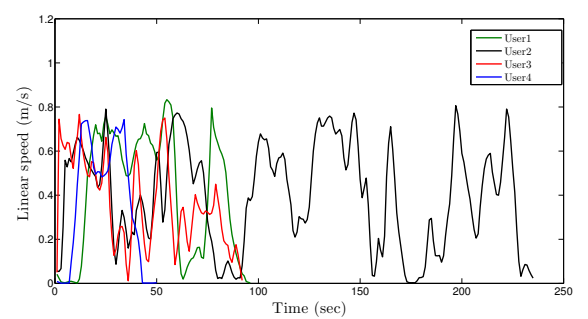

(a) Linear velocity profiles for all users while executing the Task 1 run.

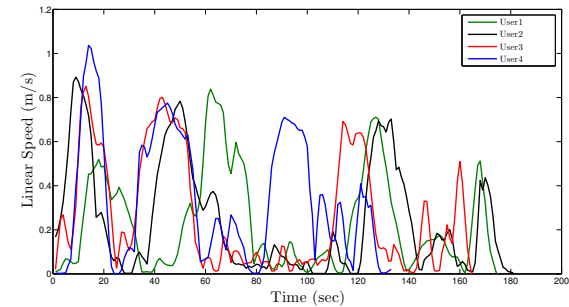

(c) Linear velocity profiles for all users while executing the Task 3 run.

Fig. 4. Linear velocity profiles for all four Task runs.

Fig. 2a shows the result of the warm up task. Fig. $2 b$ is the pose trajectory for task 2, whereas the more convoluted manoeuvre in Fig. 2c correspond to the advanced task 3, where the user was asked to drive trough two open doors (shown on the left). In the room at the top he had to manoeuvre the wheelchair to align sideways in parallel with the bed, while in the room at the bottom he had to position the wheelchair underneath a table. Finally, Fig. $2 d$ shows the last of the four runs which was a combination of all the previous ones, i.e. combining all tasks together.

Fig. 3a illustrates the specific bed alignment component of the advanced task 3 , where the right edge of the bed in the left room is shown in blue alongside the final orientation of the wheelchair. Likewise, Fig. 3b illustrates minimum distance to the door frame when the user drives the wheelchair. To demonstrate the work of the localization, zoomed figures of driving the wheelchair through the door frame are shown. Hereby the current minimum distance with the marker visualisation is illustrated.

The final metric results for all the users are collected in Table I for the advance task 3, which includes the task subcomponents scored by the OT's PIDA assessment ${ }^{2}$. It can be seen how there is high consistency in the subjective scores and objective data in the task of assessing "Distance to door". All users received higher subjective score of " 3 " and " 4 " and all were approximately $0.14 \mathrm{~m}$ from the door with little or no variation between them. A larger number of subjects is needed to determine if the one point difference in PIDA scores is statistically significantly different.

The advantage of having data from the sensor array is that the possible sub-components or parameters of a task, like

\footnotetext{
${ }^{2}$ The combination task 4 also includes these sub-components, but it could not be undertaken by all 4 users, hence was disregarded for comparison purposes
}

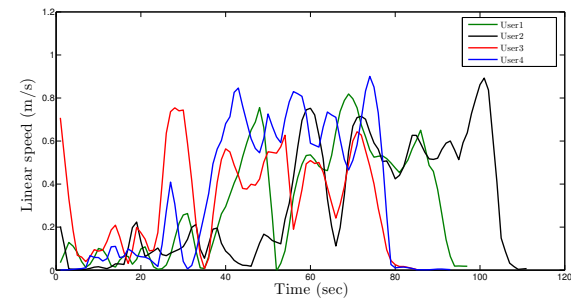

(b) Linear velocity profiles for all users while executing the Task 2 run.

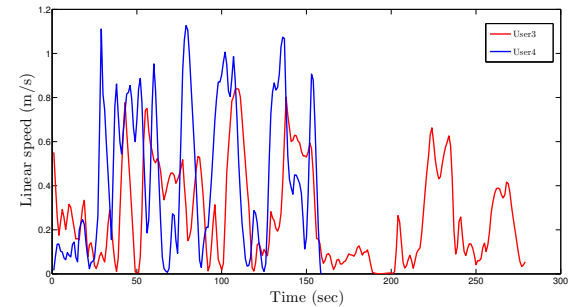

(d) Linear velocity profiles for the 2 users who undetook the Task 4 run. approaching the bed, can be studied in detail. However, it is possible that the therapist might attend to, or attribute more importance to, one particular sub-component or parameter over another. In the PIDA the task of approaching the bed is defined as "Accessing Bed - Left" or "Accessing Bed - Right". From the sensor array the subcomponents of wheelchair proximity and orientation can be determined. Users 1,2 and 4 received subjective scores of " 3 " for "Accessing Bed Right". These users were within $10 \mathrm{deg}$ of the ideal orientation to the bed and were between $0.55 \mathrm{~m}$ and $0.73 \mathrm{~m}$ from the bed. In this position it was possible for them to all touch the pillow on the bed. User 3 received a lower subjective score of "2". This user was closest to the bed $(0.45 \mathrm{~m})$ but exhibited significantly poorer orientation (18 deg error) with respect to the bed. Consequently, it is likely that the therapist was focusing on the perceived alignment discrepancy rather than distance to the bed in their subjective assessment of "Accessing Bed Right".

Fig. 4 and Fig. 5 illustrate the linear and angular velocity profiles by all four users when performing each of the four task runs. Table II shows the mean ( \pm standard deviation) of the linear and angular velocities for each user during each task. All of the data, in each sub-component of each task, was combined or lumped into a single value. Hence, there is a single linear and angular velocity result for tasks 1,2 and 3. T-tests were performed to compare each user's performance over all tasks. Users 1 and 2 significantly reduced their linear velocity when performing basic control tasks (task 2) as compared to their performance in the free trail/familiarization task (task 1). Users 3 and 4 did not significantly modify their linear velocities between tasks 1 and 2 . No user made significant changes to the angular velocity of the wheelchair between tasks 1 and 2 . User 1, 2 and 3 significantly reduced their linear and angular velocities when performing advanced control tasks (task 3) as compared to their performance in the free trail/familiarization task (task 1). User 4 did not significantly reduce either linear or 


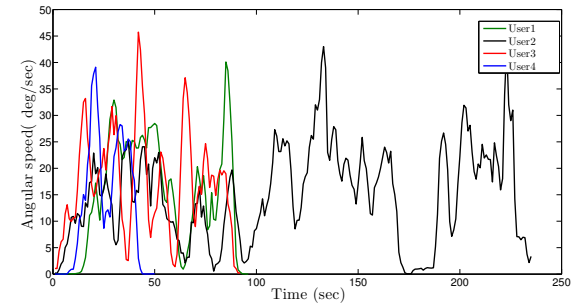

(a) Angular velocity profiles for all users while executing the Task 1 run.

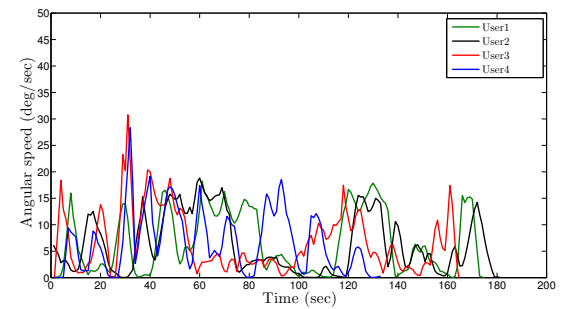

(c) Angular velocity profiles for all users while executing the Task 3 run.

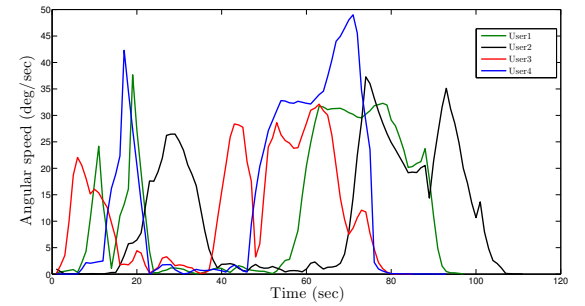

(b) Angular velocity profiles for all users while executing the Task 2 run.

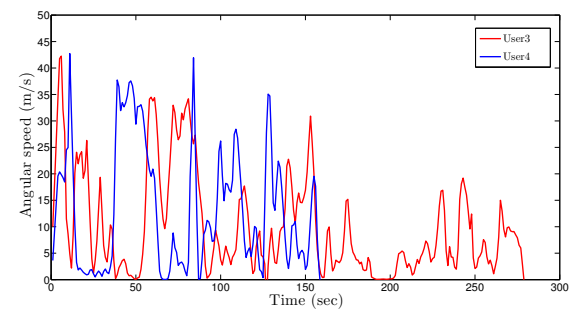

(d) Angular velocity profiles for the 2 user who undertook the Task 4 run.

Fig. 5. Angular velocity profiles for all four Task runs.

\begin{tabular}{|c||c|c|c|c|}
\hline User & $\begin{array}{c}\text { Task 1 speed } \\
\text { (linear } \pm \text { std), (angular } \pm \text { std) }\end{array}$ & $\begin{array}{c}\text { Task 2 speeds } \\
\text { (linear } \pm \text { std), (angular } \pm \text { std) }\end{array}$ & $\begin{array}{c}\text { Task 3 speeds } \\
\text { (linear } \pm \text { std), (angular } \pm \text { std) }\end{array}$ & $\begin{array}{c}\text { OT score } \\
\text { for "speed selection" }\end{array}$ \\
\hline \hline 1 & $(0.4 \pm 0.3),(14.5 \pm 14.6)$ & $(0.3 \pm 0.3),(15.9 \pm 34.7)$ & $(0.3 \pm 0.3),(7.2 \pm 7.6)$ & 4 \\
\hline 2 & $(0.4 \pm 0.3),(19.4 \pm 43.7)$ & $(0.3 \pm 0.3),(11.5 \pm 14.5)$ & $(0.2 \pm 0.3),(8.2 \pm 9.7)$ & 3 \\
\hline 3 & $(0.4 \pm 0.2),(17.1 \pm 15.4)$ & $(0.3 \pm 0.3),(13.3 \pm 15.7)$ & $(0.3 \pm 0.3),(9.1 \pm 11.2)$ & 2 \\
\hline 4 & $(0.4 \pm 0.3),(12.6 \pm 15.8)$ & $(0.3 \pm 0.4),(15.0 \pm 19.9)$ & $(0.3 \pm 0.3),(9.6 \pm 11.8)$ & 4 \\
\hline
\end{tabular}

TABLE II. WHEELCHAIR SPEEDS - LINEAR (M/SEC) AND ANGULAR ( $\%$ SEC) - AND OT SCORES OVER ALL TASKS ${ }^{3}$

angular velocity between tasks 1 and 3 . All users significantly reduced their angular velocities between tasks 2 and 3. Only User 2 also significantly reduced their linear velocities between tasks 2 and 3. Task 2 was a series of basic control activities but task 3 involved more angular movements of the wheelchair. Hence it is possible that users responded to the greater need for turns in task 3 by uniformly reducing their angular velocities.

In the PIDA, the assessor has to allocate a single score for a number of sub-components and so give an "average" score across quite different sub-components. This is especially true of "speed selection" in which the therapist is not overtly assessing either linear or angular velocity but is giving a single "average" or "integrated" value for speed linear and angular speed selection over all sub-components. User 3 perhaps responded the most appropriately by reducing speed with increasing complexity of the task and received a reasonably high subjective score of "3" for "speed selection". User 2 was the most naive wheelchair user. They significantly reduced their linear and angular velocities only in the most complex tasks (task 3) and received the lowest subjective score of "2". User 1 generally adopted strategies of reducing velocity with increasing complexity of task, as did user 3 , but was allocated a higher subjective score of "4".

\footnotetext{
${ }^{3}$ Level of significance set at $\mathrm{P} \leq 0.05$. The Bonferroni correction was not applied as it increases the likelihood of "real difference" between variables being missed [30].
}

Users 1 and 4 received the highest subjective score of "4" for speed selection. However, the speed selection strategies of each user were quite different. User 1 tended to reduce their linear and angular speed with increasing task complexity i.e. from task 1 to 3 . User 4 was the most experienced wheelchair user and only significantly reduced their angular velocity between tasks 2 and 3. Otherwise, they did not significantly modify linear or angular speed with increasing complexity of tasks. It is difficult to determine which sub-component(s) or parameter(s) the therapist was attending to when allocating the subjective score for "speed selection" in the PIDA.

Generally the therapist seems to have awarded higher subjective scores to users that significantly changed linear or angular velocity with increasing complexity of task. However, it is uncertain as to which parameters the therapist was attending to when awarding a score to user 4 . This user did not appear to significantly reduce linear or angular velocity with increasing complexity of task but still received the highest subjective rating.

\section{Discussion And Future Work}

The current study demonstrates consistency and agreement between objective data acquired from a sensing array and the subjective assessments of a therapist analysing a user's wheelchair skills. However, there was not perfect agreement between all subjective scores and data from the sensing array. 
The PIDA, like many pencil and paper assessment, does not specifically direct the therapist to attend to the specific parameters that can be derived from the sensing array. The subcomponents of the PIDA can be further resolved in more specific parameters e.g. "Accessing Bed Right" could conceivably comprise distance to bed and yaw angle with respect to bed. Similarly, "Speed selection" could refer to either (or both) linear and angular velocity but the PIDA test instructions do not stipulate which. We have demonstrated that it is likely that the therapist, covertly, attended to one or other of the parameters in scoring the PIDA. The PIDA was used for the reasons stated above i.e. it is currently in use in our facility etc. However, the intra-rater reliability coefficient of the test is low (0.67) which makes it less useful for individual comparisons [9]. This low reliability might have contributed to a reduction in consistency between subjective therapists scores and the objective data. Future developments of the project will use tests such as the "gold standard" Wheelchair Skills Test (WST-P) or WhOM etc. which have more acceptable intrarater reliability coefficients $(>0.75)$ [31].

Clinical reflection and clinical reasoning are important aspects of a therapist's professional skill development. However, there is often little or no structure as to how this complex process can be achieved [32]. The data acquired from the sensing array can definitely be used to augment the therapists observation and assessment. Having the sensor array data allows the therapist to reflect on their choice of scores and the assessment instrument i.e. the pencil and paper test they choose. Making sound, evidence based clinical decisions is becoming more critical in the area of wheelchair selection and training. The cost and complexity of wheelchairs currently being prescribed is increasing and inappropriate matches between the user and mobility devices. Concomitantly, there is higher financial burden on patients, their families and funding bodies as well as time pressures and increasingly complex decision making for therapists [33]. A therapist with access to well calibrated, accurate, reliable data from the sensing array has an opportunity to reflect on and confirm their decisions about the skill of a user and the appropriateness of their wheeled mobility device.

The more integrated data that a therapist has at their disposal the more accurate and reliable will be their assessment. A therapist might currently spend several hours with a client in performing their wheelchair assessment. However, it is not possible for the therapist to follow all their patients while they use their wheelchair both indoors and out. We have previously referred to the sensing array as a "silent therapist" [24]. The sensing array, as we have mounted it, is positioned unobtrusively behind the field of view of the user. Consequently, it is possible for the array to acquire data while the therapist is not physically present. We propose to review the possibility of acquiring longer periods of data while the user is not observed by the therapist. We did not use the information from the camera mounted in the array during the present study. However, in future developments we will consider adding the video data so that the therapist can be "telepresent", observe the environments in which the chair was used and simultaneously view data from the laser range finder and IMU to determine how the chair was managed in the observed environments. The observation could be done in real-time and streamed back to the therapists computer, or downloaded and reviewed at the therapist's discretion. The therapist could replay a user's run or trial and so have extra opportunities to reconsider and make their assessments.

Subjective assessments are biased by the experience or point of view of the assessor. Positive correlations have been reported between the WST and WST-Q. However, the WSTQ scores are often slightly higher than those of the therapist assessing the same user with the WST. The results indicate that users tend to overestimate their capacity to perform wheelchair skills as compared with the actual capacity as determined by the therapist [34]. A further development being considered is to establish a normal data base from experienced wheelchair users. An analysis package could then be developed to differentiate the performance of skilled and naive wheelchair users. The software could act as an independent first analysis option for the therapist. Software analysis systems are in routine use in electrocardiograph (EKG) machines that print a report of the heart rhythm to assist physicians in their interpretation of the data. Less experienced therapist could use the analysis packages to reflect on their own assessments of wheelchair users and determine which aspects of a less skilled wheelchair user's performance needed to be trained to improve their function. The concept of a "smart wheelchair" could then be extended to one that helps to think for the wheelchair user but also helps to think and make decisions to assist the assessing therapist. The analysis packages would thereby be employed to aid a therapist's clinical reflection and clinical reasoning around their wheelchair skills assessments.

Work is currently underway to trial the proposed solution with a wider pool of participants. Having more data available will not only help in possibly finding stronger correlations for a given pre-selected set of quantitative measures, but will also habilitate data-mining techniques in finding which quantitative measures are indeed the most informative candidates to the therapist.

\section{CONCLUSIONS}

Our study has demonstrated that accurate, reliable objective data from a sensing array and inherent algorithms can be used to augment the Occupational Therapists subjective assessment of PMD users. We found close agreement between wheelchair position and orientation to doorways and beds (in preparation for transfer from chair to bed) and to a lesser extent between estimates of wheelchair speed selection. Differences between subjective and objective data could be attributed to the wheelchair parameters to which the therapist attended i.e. distance from bed vs wheelchair orientation and linear vs angular velocity. More fundamentally, the results indicate that there is scope for therapists to reflect on their clinical reasoning and decision making in areas as diverse as choice of assessments, parameters attended to during assessments, methods of determining skill levels of PMD users and indeed, therapists themselves. There is clearly scope for therapists to guide the development of sensing technology in collaboration with engineers too. The knowledge and evidence base that therapist can thereby develop from a "silent therapist" aid such as the one proposed in this study can be critical to the future prescription of increasingly complex and pricey mobility aids. 


\section{ACKNOWLEDGMENT}

This work is based on a collaboration between the Centre for Autonomous Systems (CAS) at the University of Technology, Sydney and the Prince of Wales hospital in Sydney. The authors would like to thank Mr Peyman Biazar and Ms Janine Rudrum, from the Prince of Wales Hospital, for their assistance in the project.

\section{REFERENCES}

[1] Engineers Australia, "Policy statement - assistive technology and an ageing population," College of Biomedical Engineers, Policy Statement, 2010. [Online]. Available: http://www.engineersaustralia.org.au/da/index/getfile/id/9099

[2] P. Clarke and A. Colantonio, "Wheelchair use among community dwelling older adults: prevalence and risk factors in a national sample," Canadian Journal on Aging, vol. 24, pp. 191-198, 2005.

[3] H. S. Kaye, T. Kang, and M. P. LaPlante, Mobility Device Use in the United States. Disability Statistics Report 14. Disability Statistics Center, University of California, San Francisco, 2000.

[4] K. Huhn, P. Guarrera-Bowlby, and J. E. Deutsch, "The clinical decisionmaking process of prescribing power mobility for a child with cerebral palsy," Pediatric physical therapy : the official publication, vol. 19, pp. 254-260, 2007.

[5] F. Harris and S. Springle, "Outcomes measurement of a wheelchair intervention," Disability Rehabilitation Assistive Technology, vol. 3, pp. 171-180, 2008.

[6] R. L. Kirby, J. Swuste, D. J. Dupuis, D. A. MacLeod, and R. Monroe, "Wheelchair skills test: pilot study of a new outcome measure," Archives of Physical Medicine and Rehabilitation, vol. 83, pp. 10-18, 2002

[7] R. L. Kirby, The Wheelchair Skills Program (version 4.1), 052012. [Online]. Available: www.wheelchairskillsprogram.ca/eng/documents/ WST_Manual_version_4.1.60.pdf

[8] T. Mills, M. B. Holm, E. Trefle, M. Schmeler, S. Fitzgerald, and M. Boninger, "Development and consumer validation of the functional evaluation in a wheelchair (few) instrument," Disability and rehabilitation, vol. 24, no. 1-3, pp. 38-46, 2002.

[9] D. Dawson, R. Chan, and E. Kaiserman, "Development of the powermobility indoor driving assessment for residents of long term care facilities," Canadian Journal on Aging, vol. 61, pp. 269-276, 1994.

[10] L. Letts, D. Dawson, I. Bretholz, E. Kaiserman-Goldenstein, J. Gleason, E. McLellan, L. Norton, and C. Roth, "Reliability and validity of the power-mobility community driving assessment," Assistive Techonology, vol. 19, pp. 154-163, 2007.

[11] J. Pineau, R. West, A. Atrash, J. Villemure, and F. Routhier, "Towards a standardized test for intelligent wheelchairs," in Performance Metrics for Intelligent Systems, PerMIS 2010, 03 2010, pp. 169-174.

[12] D. Pradon, N. Pinsault, Z.Raphal, and R.Franois, "Could mobilty performance measures be used to evaluate wheelchair skills?" Journal of rehabilitation medicine official journal of the UEMS European Board of Physical and Rehabilitation Medicine, vol. 44, no. 3, pp. 276-279, 2012.

[13] F. Routhier, C. Vincent, J. Desrosiers, S. Nadeau, and C. Guerette, "Development of an obstacle course assessment of wheelchair user performance (ocawup): A content validity study." Technology and Disability, vol. 16, no. 1, pp. 19-31, 2004.

[14] S. C. Gendle, M. Richardson, J. Leeper, L. B. Hardin, J. M. Green, and P. A. Bishop, "Wheelchair-mounted accelerometers for measurement of physical activity." Disability and rehabilitation Assistive technology, vol. 7, no. 2, pp. 139-148, 2012. [Online]. Available: http://www.ncbi.nlm.nih.gov/pubmed/21967443

[15] S. E. Sonenblum, S. Sprigle, J. Caspall, and R. Lopez, "Validation of an accelerometer-based method to measure the use of manual wheelchairs.' Medical Engineering Physics, vol. 34, no. 6, pp. 781-786, 2012. [Online]. Available: http://www.ncbi.nlm.nih.gov/pubmed/22698978

[16] S. K. M. Wilson, J. P. Hasler, P. M. Dall, and M. H. M. H. Granat, "Objective assessment of mobility of the spinal cord injured in a freeliving environment." Spinal cord the official journal of the International Medical Society of Paraplegia, vol. 46, no. 5, pp. 352-357, 2008.
[17] G. U. Sorrento, P. S. Archambault, F. Routhier, D. Dessureault, and P. Boissy, "Assessment of joystick control during the performance of powered wheelchair driving tasks," Journal of NeuroEngineering and Rehabilitation, vol. 8, no. 31, pp. 1-11, 2011.

[18] C. Urdiales, M. Fernandez-Carmona, J. M. Peula, U. Cortes, R. Annichiaricco, C. Caltagirone, and F. Sandoval, "Wheelchair collaborative control for disabled users navigating indoors," Artificial Intelligence in Medicine, vol. 53, no. 3, pp. 177-191, 2011.

[19] T. Bernd, D. V. D. Pijl, and L. P. D. Witte, "Existing models and instruments for the selection of assistive technology in rehabilitation practice," Scandinavian Journal of Occupational Therapy, vol. 16, pp. 146-158, 2009.

[20] A. Souza, A. Kelleher, R. Cooper, R. A. Cooper, L. I. Iezzoni, and D. M. Collins, "Multiple sclerosis and mobility-related assistive technology: systematic review of literature," Journal of Rehabilitation Research and Development, vol. 47, no. 3, pp. 213-223, 2010.

[21] O. Fliess-Douer, Y. C. Vanlandewijck, G. L. Manor, and L. H V. D. Woude, "A systematic review of wheelchair skills tests for manual wheelchair users with a spinal cord injury: towards a standardized outcome measure," Clinical Rehabilitation, vol. 24, no. 10, pp. 867-886, 2010. [Online]. Available: http://cre.sagepub.com/content/24/10/867.abstract

[22] W. B. Mortenson, W. C. Miller, J. Boily, B. Steele, E. M. Crawford, and G. Desharnais, "Overarching principles and salient findings for inclusion in guidelines for power mobility use within residential care facilities." J Rehabil Res Dev, vol. 43, no. 2, pp. 199-208, 2006.

[23] S. K. M. Wilson, J. P. Hasler, P. M. Dall, and M. H. Granat, "Objective assessment of mobility of the spinal cord injured in a free-living environment," Spinal Cord, vol. 46, no. 5, pp. 352-357, 2008.

[24] J. V. Miro, R. Black, F. de Bruijn, and G. Dissanayake, "Semiautonomous competency assessment of powered mobility device users," International Conference on Rehabilitation Robotics, vol. 2011, pp. 16, 2011.

[25] W. Zhou, J. V. Miro, and G. Dissanayake, "Information-efficient 3D visual SLAM for unstructured domains," IEEE Transactions on Robotics, vol. 24, no. 5, pp. 1078-1087, 2008.

[26] G. Grisetti, C. Stachniss, and W. Burgard, "Improving grid-based slam with rao-blackwellized particle filters by adaptive proposals and selective resampling," Proceedings of the IEEE International Conference on Robotics and Automation, pp. 2432-2437, 2005.

[27] D. Fox, W. Burgard, F. Dellaert, and S. Thrun, "Monte carlo localization: Efficient position estimation for mobile robots," in Proceedings of the Sixteenth National Conference on Artificial Intelligence (AAAI'99), 1999, pp. 343-349.

[28] P. J. Besl and H. D. McKay, "A method for registration of 3-d shapes," IEEE Transactions on Pattern Analysis and Machine Intelligence, vol. 14, no. 2, pp. 239-256, 1992.

[29] S. Kohlbrecher, J. Meyer, O. von Stryk, and U. Klingauf, "A flexible and scalable slam system with full $3 \mathrm{~d}$ motion estimation," in Proc. IEEE International Symposium on Safety, Security and Rescue Robotics (SSRR). IEEE, 112011.

[30] M. Bland, An Introduction to Medical Statistics, ser. Oxford Medical Publications. Oxford University Press, 2000. [Online]. Available: http://books.google.com.au/books?id=_xZnQgAACAAJ

[31] W. B. Mortenson, W. C. Miller, and C. Auger, "Issues for the selection of wheelchair-specific activity and participation outcome measures: a review." Archives of Physical Medicine and Rehabilitation, vol. 89, no. 6, pp. 1177-1186, 2008.

[32] H. L. Atkinson and K. Nixon-Cave, "A tool for clinical reasoning and reflection using the international classification of functioning, disability and health (icf) framework and patient management model." Physical Therapy, vol. 91, no. 3, pp. 416-30, 2011. [Online]. Available: http://www.ncbi.nlm.nih.gov/pubmed/21273627

[33] N. Greer, M. Brasuree, and T. J. Wilt, "Wheeled mobility (wheelchair) service delivery: scope of the evidence." Annals of Internal Medicine, vol. 156, no. 2, pp. 141-146, 2012. [Online]. Available: http://www.ncbi.nlm.nih.gov/pubmed/22250145

[34] P. W. Rushton, R. L. Kirby, and W. C. Miller, "Manual wheelchair skills: objective testing versus subjective questionnaire," Archives of Physical Medicine and Rehabilitation, vol. 93, no. 12, pp. 2313-2318, 2012. 